\section{Shear rate gradients promote a bi-phasic thrombus formation on weak adhesive proteins, such as fibrinogen in a von Willebrand factor-dependent manner}

\author{
Nicolas Receveur, ${ }^{1^{*}}$ Dmitry Nechipurenko,,$^{2,3,4^{*}}$ Yannick Knapp, ${ }^{5,6}$ \\ Aleksandra Yakusheva, ${ }^{2,3,4}$ Eric Maurer, ${ }^{1}$ Cécile V. Denis, ${ }^{7}$ François Lanza, ${ }^{1}$ \\ Mikhail Panteleev, ${ }^{2,3,4}$ Christian Gachet $^{1}$ and Pierre H. Mangin ${ }^{1}$ \\ ${ }^{1}$ Université de Strasbourg, INSERM, EFS Grand-Est, BPPS UMR-S1255, FMTS, \\ Strasbourg, France; ${ }^{2}$ Faculty of Physics, Moscow State University, Moscow, Russia; \\ ${ }^{3}$ Federal Research and Clinical Centre of Pediatric Hematology, Oncology and \\ Immunology, Moscow, Russia; ${ }^{4}$ Center for Theoretical Problems of Physicochemical \\ Pharmacology, Moscow Russia; ${ }^{5} \mathrm{CNRS}$, Université Aix-Marseille, Ecole Centrale \\ Marseille, IRPHE UMR7342, Marseille, France; 'Université Avignon, LAPEC EA4278, \\ Avignon, France and ${ }^{7}$ HITh, UMR_S1176, INSERM, Université Paris-Sud, Université \\ Paris-Saclay, Le Kremlin-Bicêtre, Paris, France
}

*NR and DN contributed equally as co-first authors

\section{ABSTRACT}

$\mathrm{B}$ lood flow profoundly varies throughout the vascular tree due to its pulsatile nature and to the complex vessel geometry. While thrombus formation has been extensively studied in vitro under constant flow, and in vivo under normal blood flow conditions, increased attention has been paid to the impact of complex hemodynamics such as flow acceleration found in stenosed arteries. We investigated the effect of flow acceleration, characterized by shear rate gradients, on the function of platelets adhering to fibrinogen, a plasma protein which plays a key role in hemostais and thrombosis. While we confirmed that under constant flow, fibrinogen only supports single platelet adhesion, we observed that under shear rate gradients, this surface becomes highly thrombogenic, supporting efficient platelet aggregation leading to occlusive thrombus formation. Interestingly, this phenomenon is general as it occurs on other weak adhesive matrices including laminins and thrombospondin-1. This shear rate gradient-driven thrombosis is biphasic with an initial step of slow platelet recruitment supported by direct plasma von Willebrand factor (VWF) adsorption to immobilized fibrinogen and followed by a second phase of explosive thrombosis initiated by VWF fiber formation on platelet monolayers. In vivo experiments confirmed that shear rate gradients accelerate thrombosis in a VWF-dependent manner. Together, this study characterizes a process of plasma VWF-dependent accelerated thrombosis on weak adhesive proteins such as, fibrinogen in the presence of shear rate gradients.

\section{Introduction}

It has long been recognized that hemodynamics play important roles in hemostasis and thrombosis. ${ }^{1}$ A study coupling intravital microscopy to hydrodynamic studies provided evidence that shear gradients found at the site of vessel stenosis enhance thrombus formation in the post-stenotic area. ${ }^{2}$ The rheology-driven thrombi were essentially composed of discoid platelets as they formed independently of soluble agonists. Exacerbated platelet function and thrombosis in the poststenotic area was confirmed in another study, which proposed a role for von Willebrand factor (VWF) in this process. ${ }^{3}$ To date, the mechanism by which VWF exacerbates platelet activation and thrombosis under shear gradients has not been established. In addition to VWF, atherosclerotic plaque rupture exposes many other adhesive proteins including fibrinogen, which is known to support platelet adhesion and activation under low laminar blood flow conditions. ${ }^{4}$ However, whether

Ferrata Storti Foundation

Haematologica 2020
Volume 105(10):2471-2483

\section{Correspondence:}

PIERRE MANGIN

pierre.mangin@efs.sante.fr

Received: August 26, 2019.

Accepted: November 13, 2019.

Pre-published: November 14, 2019.

doi:10.3324/haematol.2019.235754

(C)2020 Ferrata Storti Foundation

Material published in Haematologica is covered by copyright. All rights are reserved to the Ferrata Storti Foundation. Use of published material is allowed under the following terms and conditions:

https://creativecommons.org/licenses/by-nc/4.0/legalcode. Copies of published material are allowed for personal or internal use. Sharing published material for non-commercial purposes is subject to the following conditions: https://creativecommons. org/licenses/by-nc/4.0/legalcode, sect. 3. Reproducing and sharing published material for commercial purposes is not allowed without permission in writing from the publisher. 
this protein initiates and contributes to atherothrombosis under profoundly altered flow remains unknown.

VWF is a well-studied adhesive protein for platelets that is central in both hemostasis and arterial thrombosis. ${ }^{5}$ VWF is found in the subendothelium, in Weibel-Palade bodies of endothelial cells, in $\alpha$-granules of platelets and in the plasma. Upon vascular injury, plasma VWF becomes rapidly adsorbed to several adhesive glycoproteins found at the site of injury, including collagen, laminins, thrombospondin, fibrin and fibronectin. ${ }^{6}$ Immobilized VWF then unfolds under the action of shear forces and exposes sites for the GPIb-IX-V complex supporting the recruitment of flowing platelets. VWF has the unique ability amongst all adhesive proteins to recruit platelets under elevated wall shear rate (WSR) conditions $\left.\left(>1,500 \mathrm{~s}^{-1}\right)\right)^{7-9}$ It has also been shown that unfolding of VWF is facilitated in the presence of shear gradients and elongational flow and can form a network of fibers which supports stable adhesion of several individual platelets. ${ }^{10-14}$

Fibrinogen is a soluble plasma glycoprotein which plays a pivotal role in hemostasis. In suspension, activation of platelets results in a change of the conformation of inte-
A

\section{PDMS Chamber}

\section{$3 D$ view}

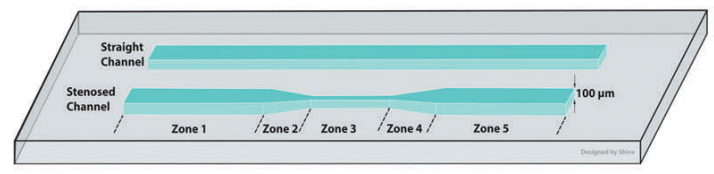

\section{Top view}

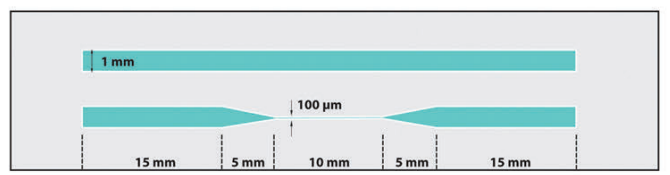

B

\section{Micro-PIV}

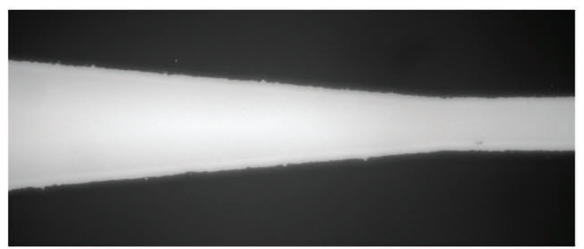

C

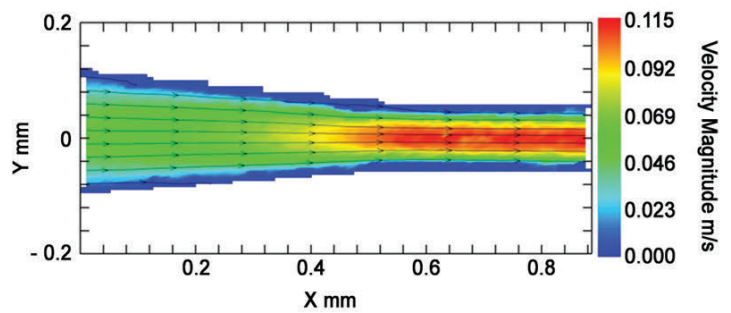

D

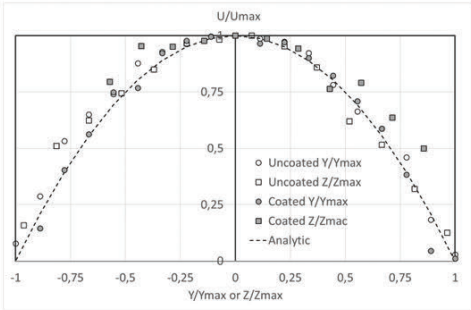

$\mathbf{F}$
E

\section{CFD}

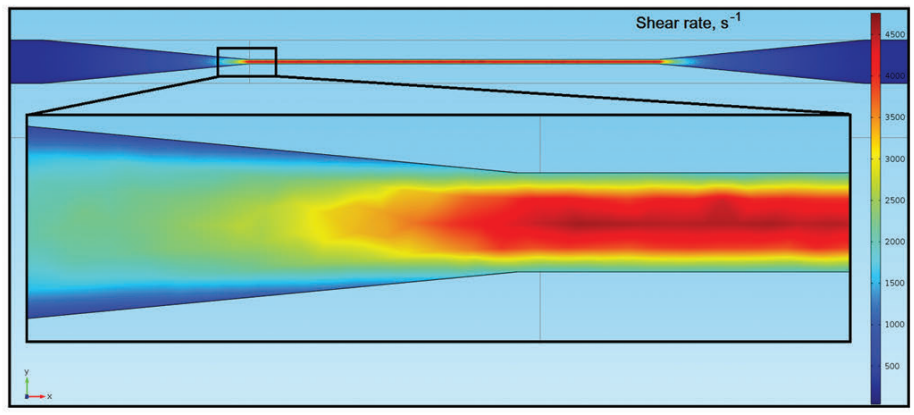

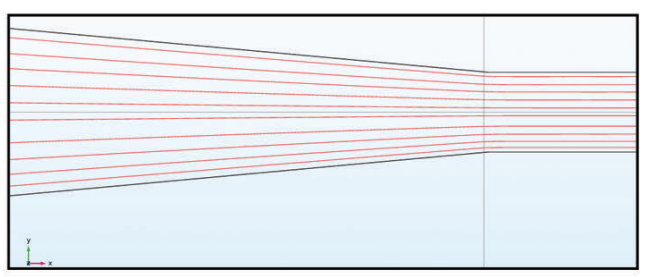

Figure 1. Schematic of a microfluidic flow chamber designed to generate shear gradients. (A) Schematic of the poly-dimethylsiloxane (PDMS)-based microfluidic flow chamber containing a straight rectangular channel with a width of $1 \mathrm{~mm}$ and a height of $0.1 \mathrm{~mm}$, and a stenosed channel with similar dimensions, but presenting a $90 \%$ reduction in width in the central region. (B) Bright field microscope view of the microfluidic flow chamber: transition from zone 2 to zone 3 of the stenosed channel. (C) Velocity magnitude heat map at mid-height in the stenosed channel obtained by micro-particle image velocimetry: transition from zone 2 to zone 3. (D) Non dimensional velocity profiles UWmax as a function of YYMmax or ZZmax ( $\mathrm{U}$ is the velocity magnitude, Ymax is the half width of the stenosis, Zmax the half height) in the mid height and mid width of the microfluidic flow chamber in zone 3. (E) Computational fluid dynamic analysis presenting the wall shear rate (WSR) heat map at the chamber floor $(\mathrm{z}=0)$ throughout the whole chamber and in the zoomed region corresponding to the entrance of zone 3 . Channel geometry in the computational fluid dynamic simulation (CFD) model corresponded to the stenosed version of the chamber shown in panel (A). (F) The streamlines for the central horizontal crosssection ( $50 \mu \mathrm{m}$ above the chamber floor) of the zone 2 to zone 3 connection region were calculated using the CFD-derived velocity field. 
A

Straight channel

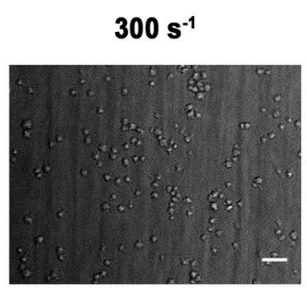

$4,800 \mathrm{~s}^{-1}$

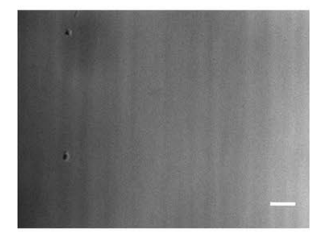

C

Stenosed channel

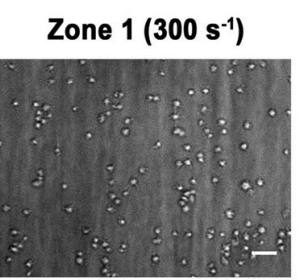

Zone $3\left(4,800 \mathrm{~s}^{-1}\right)$

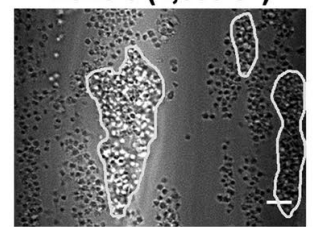

Zone $5\left(300 \mathrm{~s}^{-1}\right)$

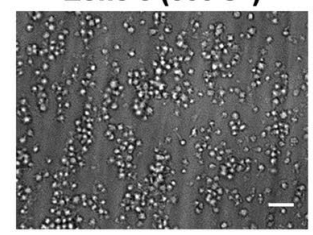

E
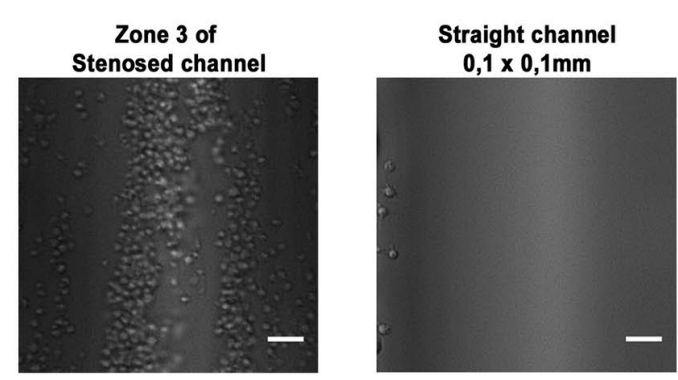

G

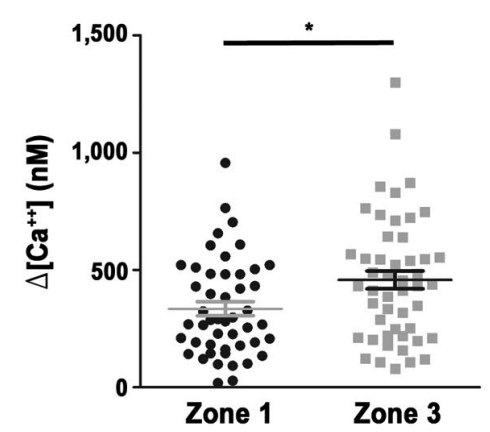

B

Straight channel

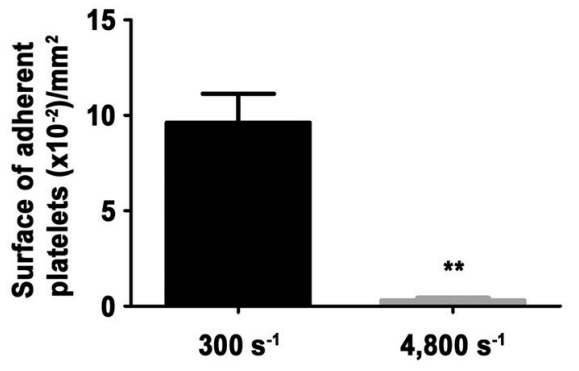

D

Stenosed channel
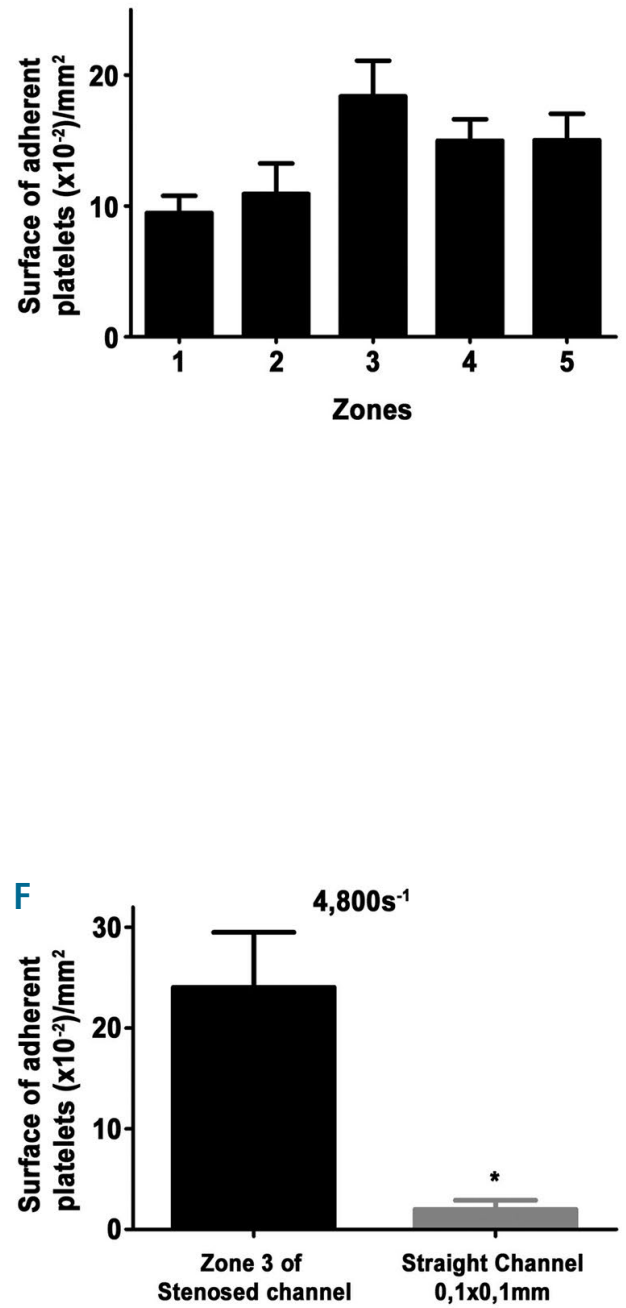

H

Zone 1

Zone 3
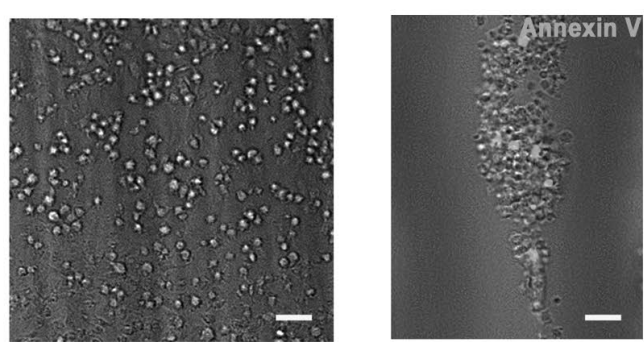
I

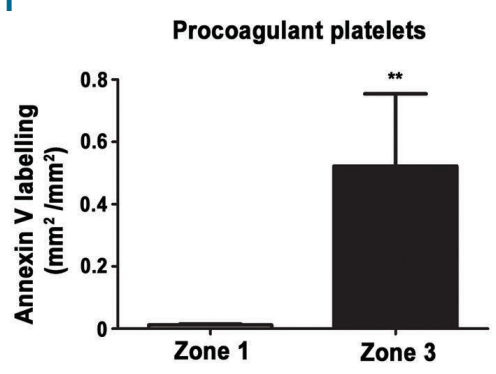

J

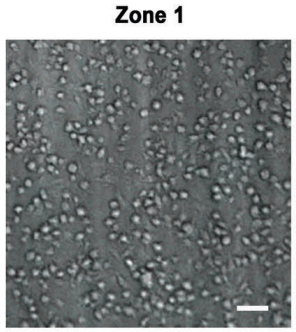

K

Zone 3

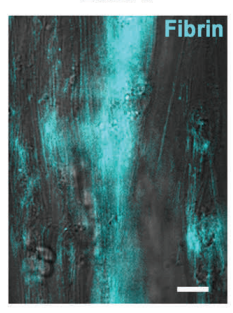

Fibrin formation

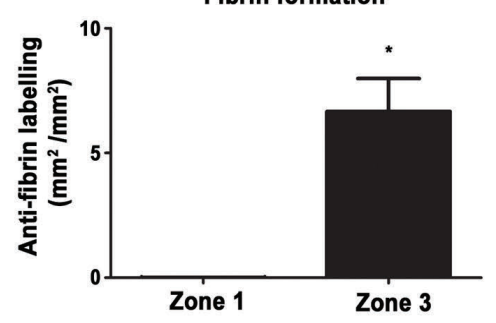

L

$2,5 \mathrm{~s}^{-1 / \mu m}$

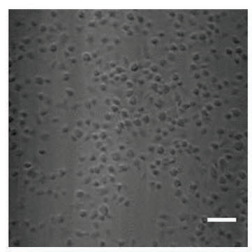

$4,9 \mathrm{~s}^{-1} / \mu \mathrm{m}$

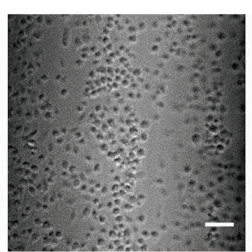

$7,4 \mathrm{~s}^{-1 / \mu \mathrm{m}}$

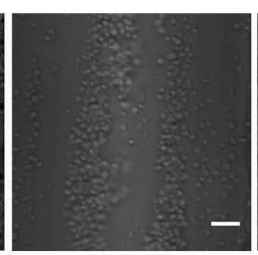

$9,8 \mathrm{~s}^{-1} / \mu \mathrm{m}$

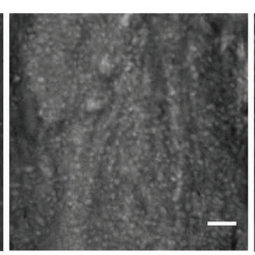

$12,2 \mathrm{~s}^{-1 / \mu \mathrm{m}}$

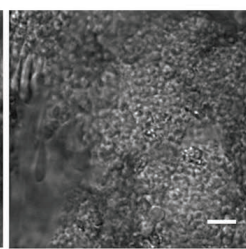

M

Stenosed Channel

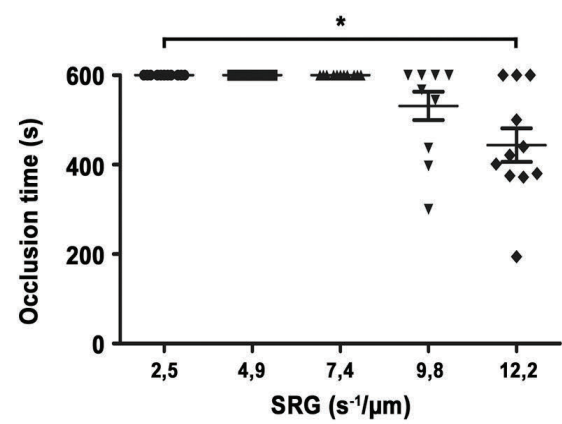

Figure 2 (previous page). Shear rate gradients promote platelet aggregation, procoagulant activity and fibrin formation on a fibrinogen surface. Hirudinated human whole blood was perfused through channels of the microfluidic chamber coated with a solution of fibrinogen $(300 \mu \mathrm{g} / \mathrm{mL})$. (A) Representative differential interfer-

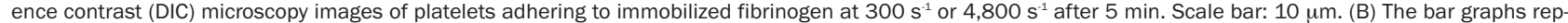
resent the surface of adherent platelets obtained after $5 \mathrm{~min}$ of perfusion. The surfaces represent the mean \pm standard error of the mean (SEM) in six random fields of five separate experiments performed with different blood donors. $* * P<0.005$. (C) Representative DIC microscopy images of platelets adhering to immobilized fibrinogen in various zones of the $90 \%$ stenosed channel, after 5 min. Scale bar: $10 \mu \mathrm{m}$. Theoretical wall shear rate (WSR) in rectangular regions were indicated. (D) The bar graphs represents the surface of adherent platelets in various regions obtained after 5 min of perfusion. The surfaces represent the mean \pm standard error of the mean (SEM) in one random field of six separate experiments performed with different blood donors. (E) Representative DIC microscopy images of platelets adhering to immobilized fibrinogen in zone 3 of the stenosed channel or in the central region of a straight rectangular channel $(0.1 \mathrm{~mm} / 0.1 \mathrm{~mm})$, at $4,800 \mathrm{~s}^{-1}$ after $5 \mathrm{~min}$. Scale bar: $10 \mu \mathrm{m}$. (F) The bar graphs represent the surface of adherent platelets obtained after 5 min of perfusion. The surfaces represent the mean \pm SEM in six random fields of three separate experiments performed with different blood donors. ${ }^{*} P<0.05$. (G) Washed human platelets loaded with morphological and Ca ${ }^{2+}$ dyes were reconstituted with $50 \%$ (vol $/ \mathrm{vol}$ ) autologous packed red blood cells at a final concentration of $2.5 \times 10^{8}$ platelets $/ \mathrm{mL}$ and perfused through the stenosed channel at $300 \mathrm{~s}^{-1}$ (in zone 1). Changes in fluorescence in platelets adhering in zone 1 and at the entrance of zone 3 were monitored for 5 min by confocal microscopy, and cytosolic $\mathrm{Ca}^{2+}$ concentrations were calculated. The dot plot of the maximal increase relative to the basal state in individual adherent platelets is shown ( $\mathrm{n}=80$ from four independent experiments). (H) Hirudinated blood was perfused over fibrinogen in the presence of Alexa 488 -conjugatdd annexin-V (1/50) at $300 \mathrm{~s}^{-1}$ for 10 min. Procoagulant platelets were detected by their annexin-V positivity (greend platelets) using a epifluorescence microscopy. Representative DIC/epifluorescence images are shown. (I) The bar graph represents the surface of annexin-V labelling per $\mathrm{mm}^{2}$ in zone 1 and zone 3 of six independent experiments, $\star \star P<0.01$. (J) Recalcified citrated human whole blood was perfused over fibrinogen $(300 \mu \mathrm{g} / \mathrm{mL})$, in the presence of the specific Alexa 647 -coupled anti-human fibrin antibody $59 \mathrm{~d} 8(5 \mu \mathrm{g} / \mathrm{mL})$. Representative DIC/fluorescence images represent fibrin formation in blue after $10 \mathrm{~min}$ of blood perfusion. (K) The bar graph represents the surface of fibrin formation per $\mathrm{mm}^{2}$, in four independent experiments. Scale bar: $10 \mu \mathrm{m}$. ${ }^{*} P<0.05$. (L) Representative DIC microscopy images of platelets accumulated on immobilized fibrinogen in zone 3 at shear rate gradients (SRG) of $2.5 \mathrm{~s}^{-1} / \mu \mathrm{m}$ (corresponding to a wall shear rate (WSR) of $1,600 \mathrm{~s}^{-1}$ ), $4.9 \mathrm{~s}^{-1} / \mathrm{um}$ (corresponding to a WSR of 3,200 s-1), $7.4 \mathrm{~s}^{-1} / \mu \mathrm{m}$ (corresponding to a WSR of $4,800 \mathrm{~s}^{-1}$ ), $9.8 \mathrm{~s}^{-1} / \mu \mathrm{m}$ (corresponding to a WSR of $6,400 \mathrm{~s}^{-1}$ ) and $12.2 \mathrm{~s}^{-1} / \mu \mathrm{m}$ (corresponding to a WSR of $8,000 \mathrm{~s}^{-1}$ ) after $10 \mathrm{~min}$ or less. Scale bar: $10 \mu \mathrm{m}$. (M) The scatter plot represent the time to occlusion in the stenosed channel and limited to the 10 first min of perfusion (the value was set at $10 \mathrm{~min}$ if no occlusion occurred). Each dot represents the value of distinct flows performed with different blood donors, * $P<0.05$.

grin $\alpha I I b \beta 3$ which supports fibrinogen binding, allowing the formation of inter-platelet bonds, i.e, platelet aggregation. ${ }^{15}$ When immobilized on a surface, fibrinogen supports attachment of resting platelets which is limited to low blood flow conditions $\left(<1,000 \mathrm{~s}^{-1}\right)$, as fibrinogen is not recognized to allow plasma VWF adsorption. ${ }^{7}$ The adhesive properties of fibrinogen under disturbed hemodynamic conditions remains completely unexplored. Understanding the effect of such flows on platelet adhe- sion to fibrinogen will help to better understand the mechanism underlying thrombosis since this pathological process is intimately linked to severely disturbed blood flow patterns.

This study evaluated the effect of shear rate gradients (SRG) such as those found at the entrance of a vessel stenosis, on platelet function on fibrinogen. A major finding is that fibrinogen becomes a highly thrombogenic surface under elevated SRG allowing the formation of occlu- 
sive thrombi. We identified and characterized a process of SRG-accelerated thrombosis that is bi-phasic with an initial step of slow platelet recruitment initiated through the ability of fibrinogen to adsorb plasma VWF, followed by a second stage during which VWF fibers of a couple of hundred microns form and initiate a very rapid accumulation of highly activated platelets to promote occlusive thrombosis. This process is general as it can be observed on other weak adhesive proteins such as laminins and thrombospondin-1.

\section{Methods}

\section{Preparation of microfluidic devices}

Microfluidic devices were made from poly-dimethylsiloxane (PDMS) by standard soft lithography techniques according to published methods. ${ }^{16}$ Briefly, a patterned photoresistant-based mold was made by standard soft lithography techniques. The liquid prepolymer PDMS and its curing agent were mixed thoroughly at a volumetric ratio of 10:1, placed in a vacuum chamber to degas and poured onto the mold followed by curing at $70^{\circ} \mathrm{C}$ for 2 hours. The polymerized PDMS channels were then carefully peeled away from the mold and holes were punched at both extremities. Finally, the irreversible bonding of PDMS to glass coverslips was performed upon exposure of both surfaces to an oxygen plasma. We made a chamber containing: $5 \mathrm{~cm}$ long straight rectangular channels with a $1 \mathrm{~mm}$ width and $0.1 \mathrm{~mm}$ height constant section (Figure 1A; straight channel) and; stenosed channels with identical dimensions in addition to a symmetric stenosis showing a $90 \%$ lumen reduction in the central part of the channel (Figure 1A; stenosed channel). This symmetric two sided stenosed channel is composed of a constricted central part (zone 3) connected on both sides to rectangular sections similar to straight channel (zones 1 and 5) through linearly varying sections (zones 2 and 4). Finally, we made a chamber containing square section channels with a width of $0.1 \mathrm{~mm}$, a height of $0.1 \mathrm{~mm}$ and a length of $5 \mathrm{~cm}$.

\section{Computational modeling of VWF dynamics}

VWF dynamics was described with a model reported in. ${ }^{14}$ In order to reduce computational complexity, hydrodynamic correlations were not taken into account. The VWF dimer was treated as a sphere with a $70 \mathrm{~nm}$ radius. The polymer chain was represented as the chain of interconnected spheres, joined with Hookean springs. The interaction parameters were taken from. ${ }^{14}$ In order to infer conformational dynamics of VWF molecules within different flow lines of the chamber (flow lines lying within the central z-x plane were considered), the corresponding flow velocity profiles were taken from the computational fluid dynamic (CFD) simulation results. The local velocity gradient tensor was computed from polynomial interpolation of velocity profiles obtained from CFD simulations. In order to reduce the computational burden we considered VWF dynamics along the $1 \mathrm{~mm}$ of flow lines corresponding to stenosis entrance region (as far as this region possesses the highest values of elongational velocity gradients). VWF elongation $d$ for a given moment of time was determined as maximum linear size of VWF molecule divided by the length of fully unfolded molecule. Values of VWF elongation exceeding unity correspond to flow-mediated extension of linear unfolded molecule. In case VWF elongation exceeded unity, the molecule was considered to be fully unfolded. In order to obtain a relative value of unfolding events we considered flow lines with different heights above fibrinogen surface: $1,5,10,15$ and $20 \mu \mathrm{m}$. For each height above the surface, 100 VWF trajectories were analyzed to infer the mean relative number of VWF unfolding events. The maximum relative number of unfolding events were analyzed for each flow line. Analysis was performed for long ( $\mathrm{n}=80$ dimers) VWF multimers.

\section{In vivo $\mathrm{FeCl}_{3}$ thrombosis and stenosis}

$\mathrm{FeCl}_{3}$-induced thrombosis was performed as previously described ${ }^{17-19}$ with some modifications. Briefly, 8-10 week-old mice were anesthetized by intraperitoneal injection of a mixture of xylasine $(100 \mathrm{mg} / \mathrm{kg})$ and ketamine $(20 \mathrm{mg} / \mathrm{kg})$. DiOC 6 ( $0.4 \mu \mathrm{L}$ of a $100 \mu \mathrm{M}$ solution/g of body weight) was injected into the jugular vein to label the thrombus. The carotid artery was injured by lateral external application of a filter paper containing a $4 \% \mathrm{FeCl}_{3}$ solution for 2.5 minutes ( $\mathrm{min}$ ). Four min after vessel injury, a 80\% stenosis of the carotid or no stenosis (control) was obtained by compression with a micro-manipulator (Eppendorf Transferman, Hamburg, Germany). Thrombus formation was monitored with a fluorescent macroscope (Macrofluo ${ }^{\varpi}$, Leica Microsystems, RueilMalmaison, France). Ethics approval for animal experimentation was obtained from the French Ministry of Research in accordance with the European Union Guidelines as defined by European laws.

Detailed methods are described in the Online Supplementary Materials and Methods.

\section{Results}

\section{Development and characterization of a microfluidic device to study the effect of SRG on platelet adhesion to fibrinogen}

We developed a PDMS-based microfluidic chamber with a $90 \%$ narrowing in its central region (zone 3) in order to generate SRG, similar to those found in pathological settings (Figure 1A). Micro-particle imaging velocimetry (microPIV) measurements performed while perfusing a 40/60 glycerol/PBS mixture to mimic the blood viscosity, at a flow rate of $30 \mu \mathrm{L} / \mathrm{min}$, showed terminal acceleration of the flow at the end of zone 2 and the entry of the stenosis (zone 3), (Figure 1B-C). The maximal flow velocity of $0.115 \mathrm{~m} / \mathrm{s}$ measured in the centerline of the stenotic area (Figure $1 \mathrm{C}$ ) is very close to the expected $0.113 \mathrm{~m} / \mathrm{s} \mathrm{com-}$ puted from the mass flow rate conservation through the model and to that of the straight channel (data not shown). In agreement, the value of the absolute average of WSR $\left(4,750 \pm 300 \mathrm{~s}^{-1}\right)$ of zone 3 is also very close to the $4,800 \mathrm{~s}^{-1}$ for which the stenosed channel was designed. Reynolds numbers (Re) based on maximum centreline velocity and hydraulic diameter were characteristic of a laminar flow in all sections of the flow chamber (zone 1-5: $\operatorname{Re}=0.46$; zone $3: \operatorname{Re}=3.36$ ) indicating the absence of turbulence. This laminar flow behavior was confirmed by the velocity profiles that can be inferred from the flow field measurements in the different sections of zone 3 (Figure 1D). In agreement with the microPIV measurements, computational fluid dynamic analysis indicated that the WSR were symmetrically distributed within the stenosed channel and of similar values (for $30 \mu \mathrm{L} / \mathrm{min}$ : zone 3 of 4,800 s s. $^{-1}$ (Figure 1E-F). Finally, analytical solution of Navier-Stokes equation for infinitely long squareshaped channels ${ }^{20}$ with a corresponding volumetric flow rate gives a WSR value in the center line of zone 3 of 4,792 $\mathrm{s}^{-1}$, which is in line with CFD and microPIV results.

\section{Effect of SRG on the function of platelet adhering to fibrinogen and other weak adhesive proteins}

In agreement with previous reports, ${ }^{7}$ perfusion of hirudinated whole blood over immobilized fibrinogen in 
channels with a constant section results in a modest platelet adhesion under low WSR $\left(300 \mathrm{~s}^{-1}\right)$, and no platelet recruitment under high WSR $\left(4,800 \mathrm{~s}^{-1}\right)$, (Figure $\left.2 \mathrm{~A}-\mathrm{B}\right)$. In sharp contrast, in zone 3 of the $90 \%$ narrowed channel, we observed that numerous platelets adhered at 4,800 s-1, and formed aggregates (Figure 2C-D). Control experiments indicated that this adhesion was specific as no platelet adhesion was observed in any region of the channel in the absence of coating or when the chamber was passivated with albumin (data not shown). The efficient platelet accumulation on fibrinogen in zone 3 did not rely on local geometry, since no platelet adhesion was observed at similar WSR $\left(4,800 \mathrm{~s}^{-1}\right)$ in a straight fibrinogen coated channel with identical dimensions as those of zone 3 (Online Supplementary Figure S1 and Figure 2E-F). We therefore concluded that the efficient adhesion on fibrinogen at high WSR $\left(4,800 \mathrm{~s}^{-1}\right)$ observed in zone 3 , results from flow acceleration occurring upstream (exit of zone 2) which generates SRG (Figure 1C). At early time points, we observed a modest $34.3 \%$ increase in $\mathrm{Ca}^{2+}$ signaling of platelets adhering to zone 3 compared to those attaching in zone 1 (Figure 2G). In contrast, at later stages, numerous platelets were Annexin-V positive in zone $3\left(4,800 \mathrm{~s}^{-1}\right)$, but not in zone $1\left(300 \mathrm{~s}^{-1}\right)$, suggesting that the level of platelet activation increases over time and that SRG favor procoagulant platelet formation (Figure $2 \mathrm{H}-\mathrm{I}$ ). In agreement, a signal for fibrin was detected in zone 3 when recalcified citrated human blood was perfused (Figure 2J-K). When different SRG were used, we observed single platelets adhering at $2.5 \mathrm{~s}^{-1} / \mathrm{m}$ and $4.9 \mathrm{~s}^{-1} / \mathrm{m}$, platelet aggregates started to form at $7.4 \mathrm{~s}^{-1} / \mathrm{m}$ and became massive resulting in channel occlusion at $9.8 \mathrm{~s}^{-1} / \mathrm{m}$ and $12.2 \mathrm{~s}^{-1} / \mathrm{m}$ (Figure 2L$\mathrm{M}$ and Online Supplementary Video S1). Of importance, this phenomenon appears general as it also occurred on other weak adhesive proteins including vascular laminins and thrombospondin-1 (Online Supplementary Figure S2). Together, these results indicate that SRG can enhance platelet function on weak adhesive proteins, such as fibrinogen resulting in occlusive thrombosis.

\section{Thrombus formation on fibrinogen under SRG is bi-phasic}

To further characterize the SRG-mediated process of platelet aggregation on fibrinogen, video-microscopy experiments were conducted. Real-time analysis of the flow experiments revealed a stepwise process of in vitro thrombus formation on fibrinogen, with an initial phase characterized by a slow and heterogeneous recruitment of individual platelets, followed by a stage of very rapid platelet aggregation visualized after 180 seconds (s) (Online Supplementary Video S2 and Figure 3A). Importantly, platelet adhesion and thrombus growth occur at the entrance of zone 3. Quantification of the surface coverage confirmed the bi-phasic nature of platelet accumulation on fibrinogen in zone 3 (Figure $3 \mathrm{~B}$ ). This bi-phasic process contrasted with platelet adhesion in zone 1 within the same channel which was linear (Figure 3B). The second phase (180-360 s) led to a very marked acceleration of platelet accumulation,
A
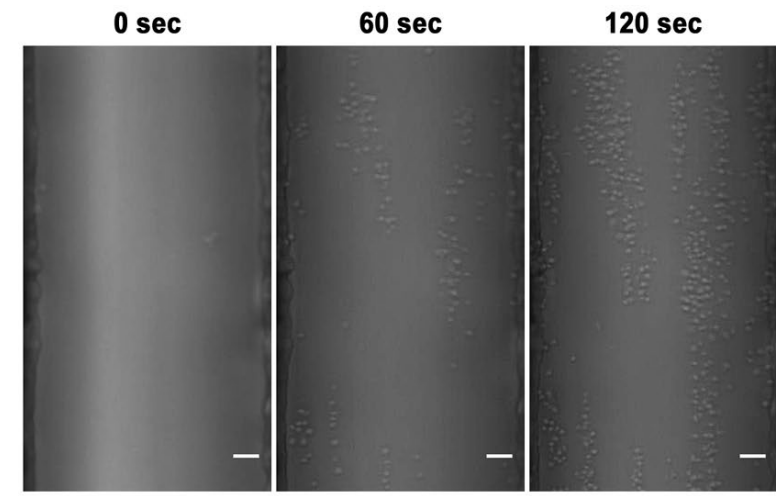

B

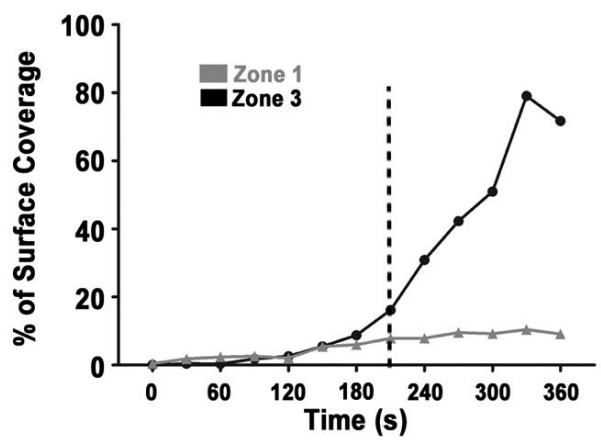

$180 \mathrm{sec}$

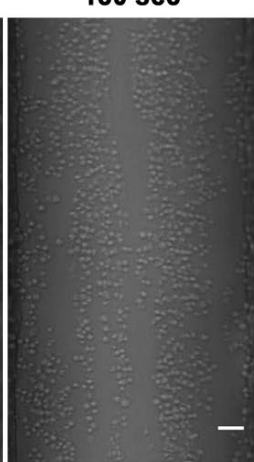

$240 \mathrm{sec}$
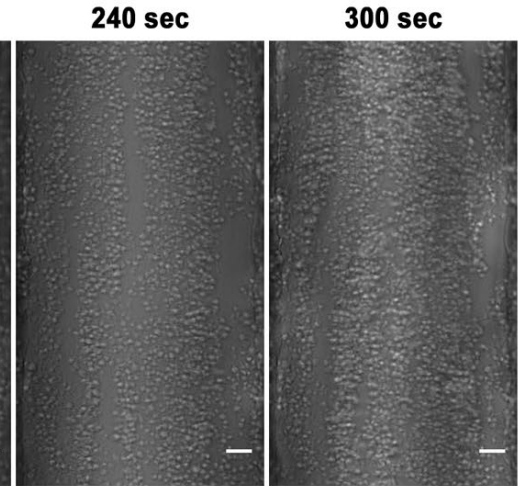

C

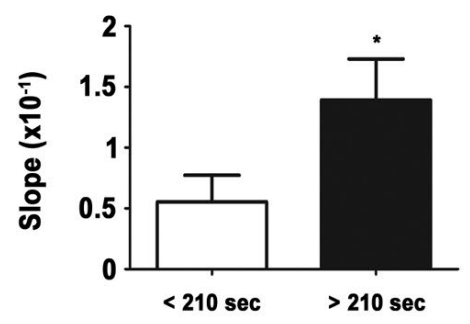

Figure 3. Platelet accumulation in the stenosed region under shear rate gradients (SRG) is bi-phasic. Hirudinated human whole bloos was perfused through channels of microfluidic chambers coated with a fibrinogen solution (300 $\mu \mathrm{g} / \mathrm{mL})$. (A) Representative differential interference contrast (DIC) microscopy images of platelets accumulating on immobilized fibrinogen in the stenosed region at indicated time points. Scale bar: $10 \mu \mathrm{m}$. (B) Representative curves of platelet accumulation in zone 1 and zone 3 over time. (C) The bar graph represents the slope of platelet accumulation in zone 3 before and after 210 s. Values are the mean \pm standard error of the mean (SEM) in eight separate experiments, $* P<0.05$. 
A

Straight Channel

$300 \mathrm{~s}^{-1}$

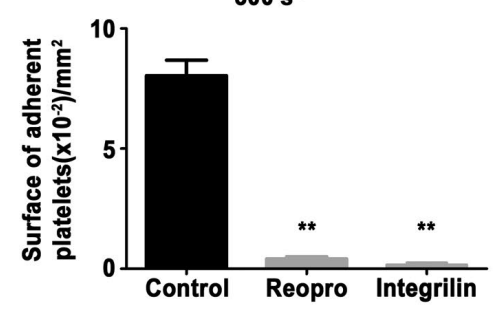

D

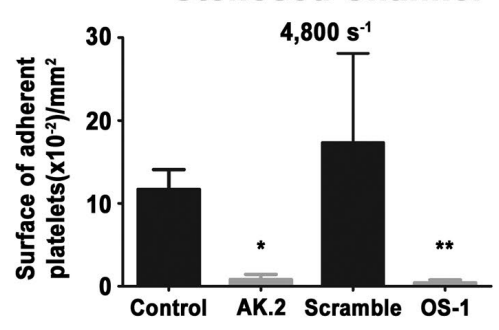

B

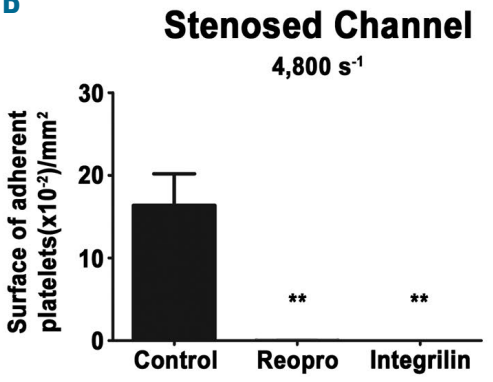

E Straight Channel

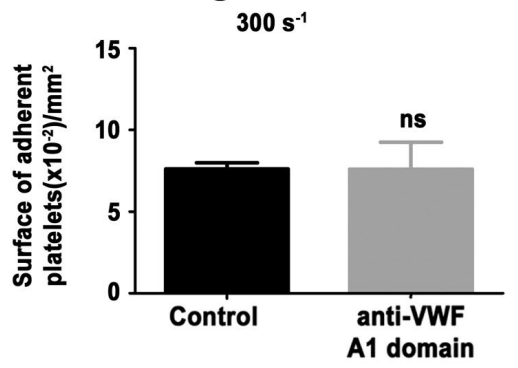

C Straight Channel

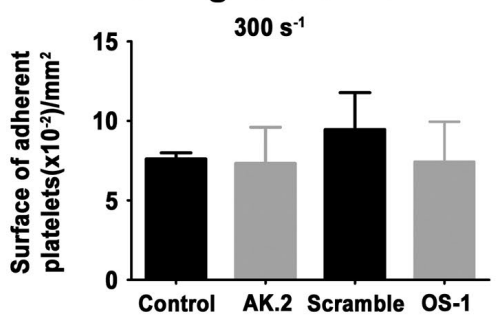

F

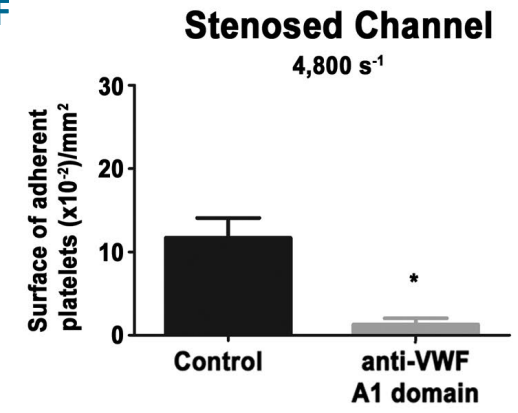

G

HSA
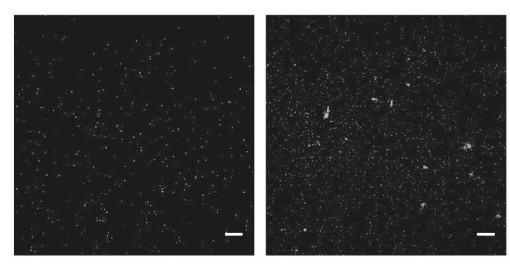

Zone 3

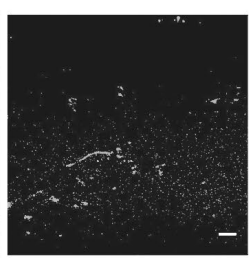

H

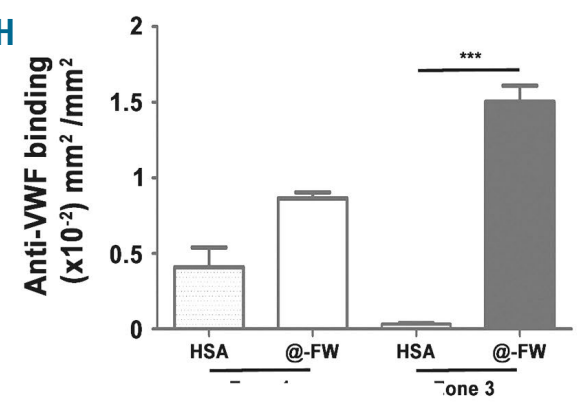

I

J
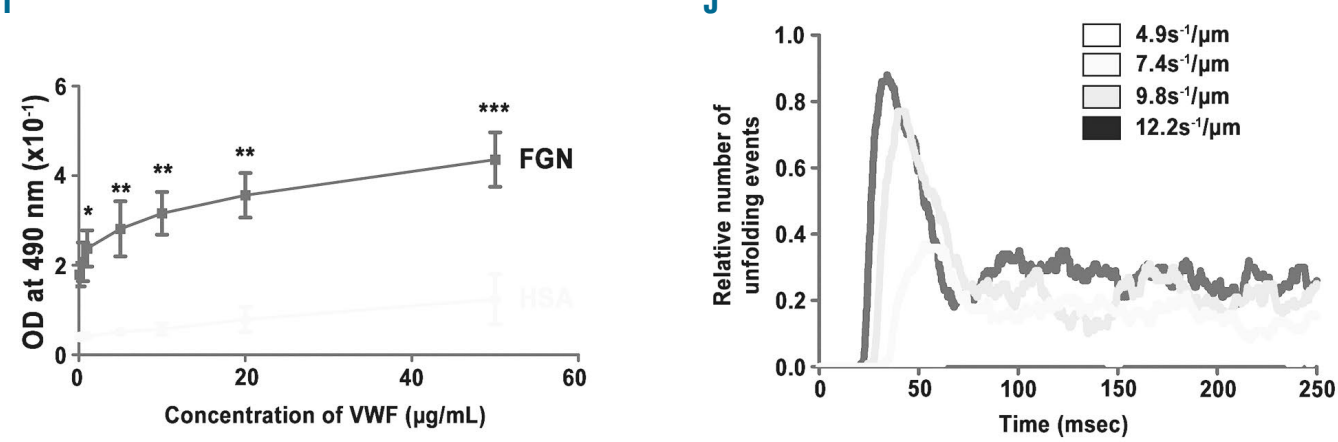

K

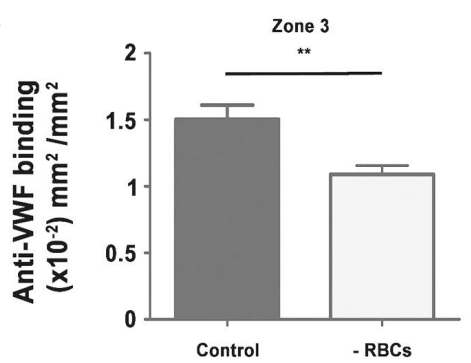

Figure 4 (previous page). Integrin $\alpha$ llb $\beta 3$ flow and the GPIb/VWF axis support platelet adhesion under shear rate gradients (SRG). Hirudinated human whole blood was perfused through channels of microfluidic chambers coated with a fibrinogen solution $(300 \mu \mathrm{g} / \mathrm{mL})$ at indicated local wall shear rates. The blood was incubated with the anti- $\alpha$ llb $\beta 3$ blockers, Reopro: $40 \mu \mathrm{g} / \mathrm{mL}$ or Integrilin: $40 \mu \mathrm{g} / \mathrm{mL}$ (A-B), the blocking anti-GPlb $\alpha$ antibody AK2 $(10 \mu \mathrm{g} / \mathrm{mL}),(C-D)$, the antiGPIb $\alpha$ peptide OS-1 (800 nM), (C-D), the anti-VWF A1 domain antibody $701(20 \mu \mathrm{g} / \mathrm{mL})$, (E-F), or with PBS (A-B), an irrelevant antibody (C-F), or a scrambled peptide (C-D) as a control. The bar graph represents the surface of adherent platelets obtained after 5 min of perfusion. The surfaces represent the mean \pm standard error of the mean (SEM) in six random field of four to six separate experiments performed with different blood donors. $* P<0.05, * * P<0.01$. (G) Representative immunofluorescence images of zone 1 and zone 3 depicting von Willebrand factor (VWF) labelling after perfusion of platelet-poor plasma (PPP) reconstituted with red blood cells (RBC) over immobilized fibrinogen (300 $\mu \mathrm{g} / \mathrm{mL})$ or human serum albumin $(\mathrm{HSA}),(300 \mu \mathrm{g} / \mathrm{mL})$ within the channels of the microfluidic chambers at $300 \mathrm{~s}^{-1}$. (H) The bar graph represents the surface of VWF labelling per $\mathrm{mm}^{2}$ on fibrinogen or HSA after 5 min of perfusion in zone 1 or zone 3 . $* * * P<0.001$. (I) Microwells were coated overnight at $4{ }^{\circ} \mathrm{C}$ with fibrinogen or HSA at $10 \mu \mathrm{g} / \mathrm{mL}$. Increasing concentrations of purified VWF were then added, and bound VWF was detected with a peroxydase-conjugated anti-VWF antibody. Curves show the optical density (OD) at $490 \mathrm{~nm}$, and values are the mean \pm SEM in three separate experiments performed in duplicate. (J) Prediction model for the relative number of VWF unfolding events in entrance of zone 3 under indicated SRG. (K) PPP was mixed or not with RBC and perfused through stenosed channels of microfluidic chambers coated with fibrinogen $(300 \mu \mathrm{g} / \mathrm{mL})$ at $300 \mathrm{~s}^{-1}$. The channels were labeled with an anti-VWF antibody. The bar graph represents the surface of VWF staining per $\mathrm{mm}^{2}$ in zone 3 after 5 min of perfusion. $* * P<0.01$. 
A

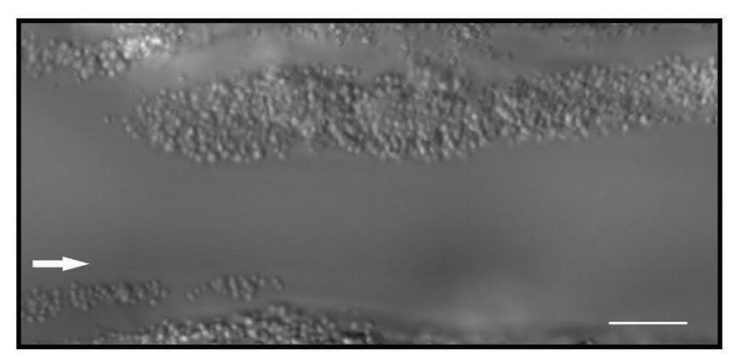

B

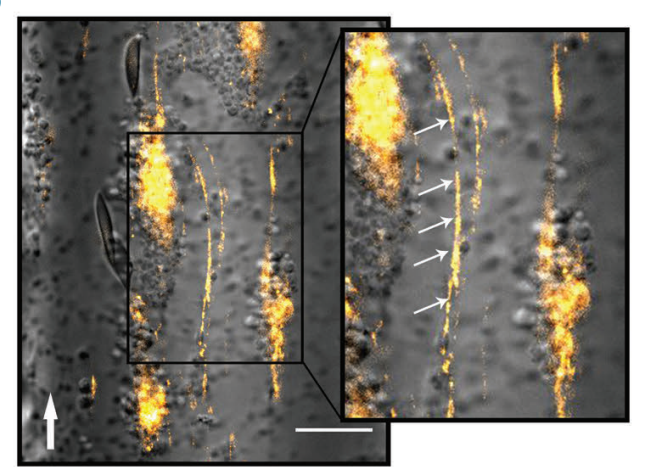

C
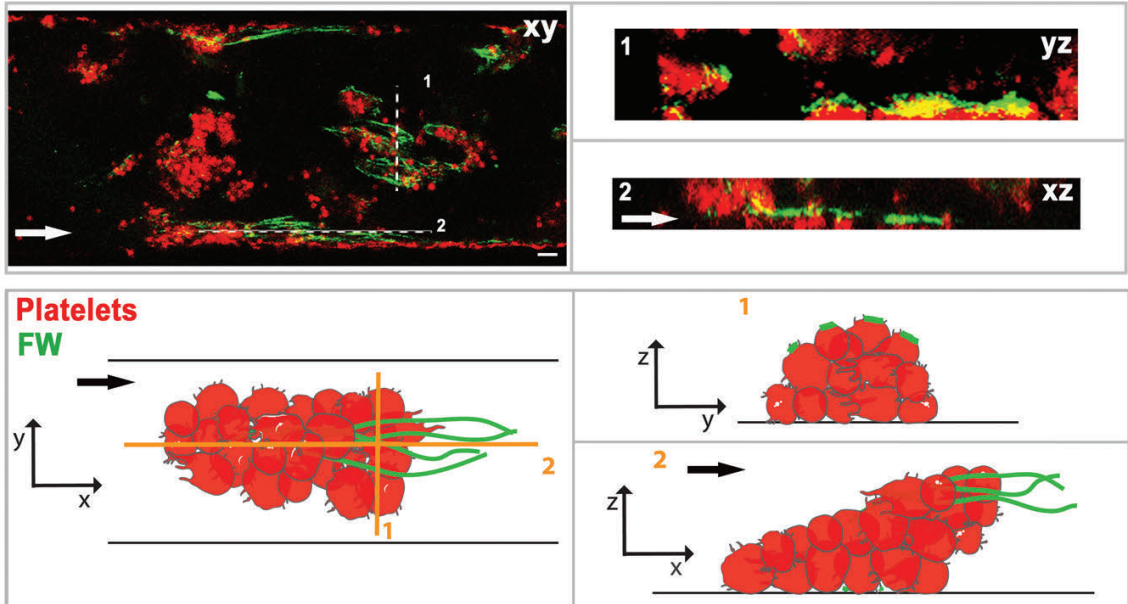

D

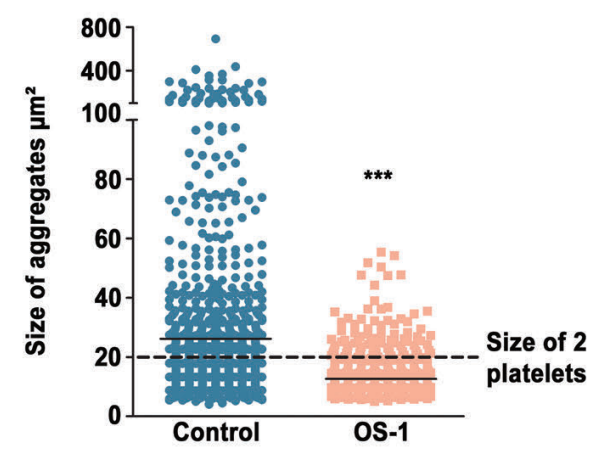

E

F

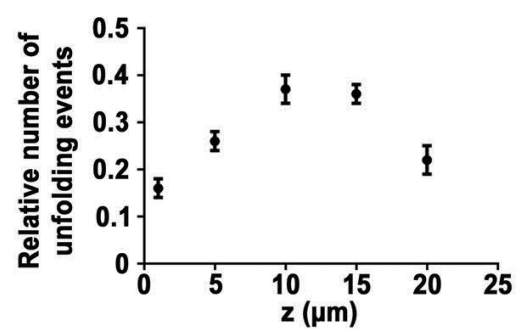

G

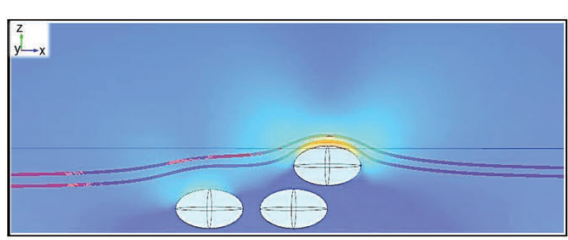

H

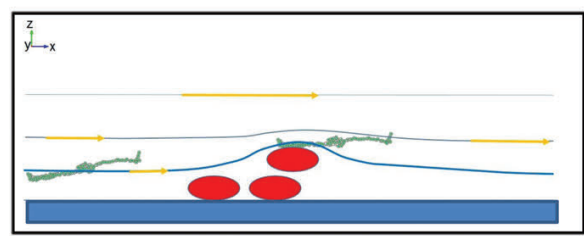


Figure 5 (previous page). The enhanced platelet aggregation under shear rate gradients occurring on fibrinogen results from plasma von Willebrand factor fiber formation. Hirudinated human whole blood was perfused through channels of the microfluidic flow chamber coated with a solution of fibrinogen ( $300 \mathrm{\mu g} / \mathrm{mL}$ ). (A) Representative differential interference contrast (DIC) microscopy image obtained in zone 3. Scale bar: $20 \mu \mathrm{m}$. (B) Representative DIC/fluorescent images represent the platelet aggregates (DIC) and VWF fibers (orange) formed in zone 3 following shear rate gradients (SRG). Scale bar: 20 um. (C) Representative confocal images and schematic of platelet aggregates formed in zone 3. Platelets appear in red (RAM.1-Cy3 at $2 \mu \mathrm{g} / \mathrm{mL}$ ), von Willebrand factor (VWF) in green (anti-VWF-Alexa488 at $2 \mu \mathrm{g} / \mathrm{mL}$ ), and the overlay in yellow. Scale bar: $10 \mu \mathrm{m}$. Schematic of platelet aggregates (red) and VWF fibers (green) formed in zone 3 following SRG. (D) Dot plot represents the size of aggregates formed in presence or absence of OS-1 that was perfused once a platelet monolayer on fibrinogen had already formed. The shading corresponds to the standard error of mean (SEM). Data were compared by 2-tailed Mann-Whitney tests, $* P<0.0001$. (E) Computational fluid dynamic (CFD)-derived heatmap of shear rate values calculated for the system of three adhered platelets in the stenosed part of the chamber. The central section of system is shown Platelets were placed in the central z-x plane of the stenosed part of chamber, near the wall. Hydrodynamic conditions are the same as described in Figure 1E. (F) The dependence of maximum relative number of VWF unfolding events on the height above the fibrinogen surface. Computational modeling of VWF dynamics was performed using the CFD-derived values of shear and elongation rates at different heights above the surface. For each height 100 simulations of long VWF multimers dynamics were obtained. Given values correspond to zone 2-zone3 connection, where VWF unfolding was maximal (see peaks at Figure 4J). VWF molecule was considered unfolded only if its maximal size exceeded contour length of the multimer. Data correspond to the same hydrodynamic conditions as used for Figure $1 \mathrm{E}$ and Figure $5 \mathrm{E}$ (maximal SRG of $7.4 \mathrm{~s}^{-1} / \mu \mathrm{m}$ ). (G) The effect of flow line contraction illustrated with CFD simulation for system of three ellipsoidal platelets. The flow lines are shown in dark red color. Note the convergence of flow lines above the platelets. (H) Schematic illustration of the consequence of flow line contraction effect: objects of finite size (like flowing VWF depicted in the figure or platelet) traveling at some distance above the surface will eventually interact with the obstacle (platelet aggregate) due to flow line convergence above the obstacle (platelet) leading to much closer distance between the flowing object and the barrier (platelet).

with a 3-fold slope increase when compared to early time points $(<210 \mathrm{~s}$ ) (Figure 3B-C). This bi-phasic platelet accumulation is original since platelet aggregation under flow is recognized to be linear over a very wide range of WSR in the classical model of "in vitro thrombus formation" when whole blood is perfused over collagen (data not shown).

\section{Characterization of the first step of platelet adhesion to fibrinogen in the stenosed region}

We next analyzed the mechanism involved in platelet adhesion to fibrinogen in zone 3. Two clinically used $\alpha I$ II b3 blockers, ReoPro ${ }^{\circledR}$ and Integrilin ${ }^{\circledR}$, completely prevented platelet adhesion in the straight control channel at $300 \mathrm{~s}^{-1}$, as well as in zone 3 of the stenosed channel $(4,800$ $\mathrm{s}^{-1}$ ), (Figure 4A-B). Blockade of GPIb $\alpha$ or VWF exhibited a similar effect in zone 3 , but not in the control channel at 300 $\mathrm{s}^{-1}$ (Figure 4C-F). These results suggested that plasma VWF becomes adsorbed to fibrinogen under SRG. This was confirmed by VWF immunostaining in zone 3 after perfusion of platelet-poor plasma (PPP) through the channel (Figure 4G). VWF staining was also detected in zone 1 but the signal was much weaker when compared to zone 3 further demonstrating that VWF binds to fibrinogen (Figure $4 \mathrm{H}$ ). Direct interaction between VWF and fibrinogen was demonstrated in a purified assay under static conditions showing a specific and dose-dependent binding of soluble VWF to immobilized fibrinogen (Figure 4I). The increased VWF adsorption observed in zone 3 might be the result of VWF unfolding. A computational modeling approach of VWF dynamics, indicated that SRG similar to those occurring in transition from zone 2 to zone 3 , especially $9.8 \mathrm{~s}^{-1} / \mathrm{m}$ and $12.2 \mathrm{~s}^{-1} / \mathrm{m}$, favored unfolding of VWF molecules (Figure 4J and Online Supplementary Video S3), while this was not the case under flow conditions found in zone 1 at $300 \mathrm{~s}^{-1}$ and zone 3 at 4,800 $\mathrm{s}^{-1}$ (Online Supplementary Figure S3). We observed a $27.5 \%$ reduction of VWF deposition in zone 3 when PPP was perfused in the absence of red blood cells, suggesting that these cells contribute to this process (Figure $4 \mathrm{~K})$. Together, these results demonstrate that platelet adhesion observed on fibrinogen in zone 3 relies on integrin $\alpha I I b \beta 3$, the GPIb-IX complex, and on plasma VWF adsorption which is the result of its unfolding due to SRG.

\section{Characterization of the second step of accelerated platelet aggregation in the stenosed region: a role for VWF fibers}

Real-time video-microscopy showed that aggregates presented an unusual extended shape in the flow direction and contained VWF fibers (Figure 5A-B and Online Supplementary Video S2 and S4). The fibers were particularly long reaching several hundred microns. Confocal microscopy confirmed the presence of VWF fibers and showed that they did not form directly on the fibrinogen surface but rather on top of platelet monolayers (Figure 5C). Interestingly these fibers appeared concomitantly to the initiation of the accelerated thrombosis, suggesting a role for them in this process (Online Supplementary Video S4). This role was confirmed in an experiment in which blockade of the GPIba/VWF axis with OS-1, once a platelet monolayer on fibrinogen had already formed, prevented the second phase of accelerated thrombosis (Figure 5D). Computational fluid dynamic simulation (CFD) of the flow around single adhered platelets in zone 3 demonstrates a very strong increase in shear at their surface $\left(>10,000 \mathrm{~s}^{-1}\right)$, suggesting that events of VWF unfolding are very likely to occur (Figure $5 \mathrm{E}$ ). Moreover, computational analysis of VWF dynamics at different heights shows SRG-dependent increase of VWF unfolding with an increase of height above the fibrinogen surface, reaching a maximum at $10 \mu \mathrm{m}$ above the surface (Figure 5F). This result provides an explanation for acceleration of thrombus growth due to SRG-mediated unfolding of VWF molecules, which might trigger VWF fiber formation. Besides, CFD also shows significant streamline contraction effects on a second layer of platelets (Figure 5G) which might be responsible for the observed increase in the rate of VWF and platelet transport to the surface of growing aggregates as illustrated in Figure $5 \mathrm{H}$. Thus, enhanced VWF unfolding and fiber formation combined with streamline contraction effects might explain the observed acceleration of thrombus formation in zone 3.

\section{SRG accelerate in vivo thrombus growth in a VWF-dependent manner}

To evaluate whether SRG accelerate thrombus growth in vivo, an experimental thrombosis approach was used. A $4 \% \mathrm{FeCl}_{3}$ solution was applied to the carotid artery of adult mice to injure the vessel wall, and once a monolayer of platelets had formed ( $270 \mathrm{~s})$, the artery was severely stenosed $(>80 \%)$ with a micromanipulator to generate SRG. Measurements of blood flows in the mouse carotid with a Doppler probe combined to CFD analysis indicated that the SRG generated in such a setting were very similar to those we applied in vitro as they were reaching $8.6 \mathrm{~s}^{-1} / \mathrm{m}$ (Figure 6A). Intravital microscopy showed that thrombus formation occurred much earlier in a stenosed vessel com- 
pared to the control (Figure $6 \mathrm{~B}$ and Online Supplementary Video S5). Quantification confirmed that thrombus formation was more rapid in the presence of a stenosis (Figure 6C-D). As expected thrombus formation was severely impaired in VWF-deficient mice under normal conditions
(Figure 6E-F). Interestingly, even though a tendency of increased thrombosis could be observed in the presence of a stenosis in $\mathrm{VWF}^{-/}$mice, no significant difference in the time of thrombus formation was observed in these mice in the presence or absence of a stenosis (Figure 6E-F). This
A

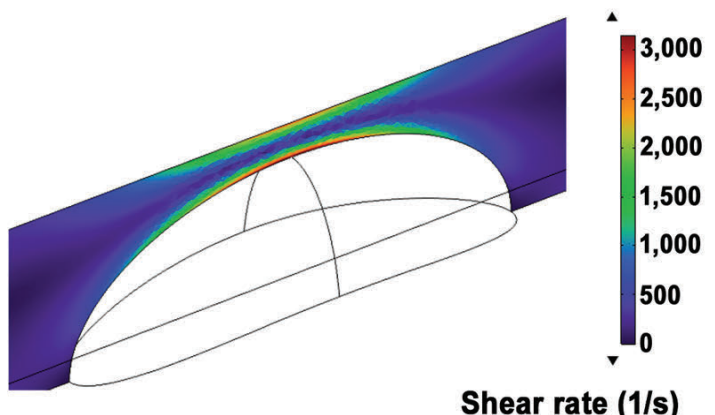

Shear rate $(1 / s)$
B
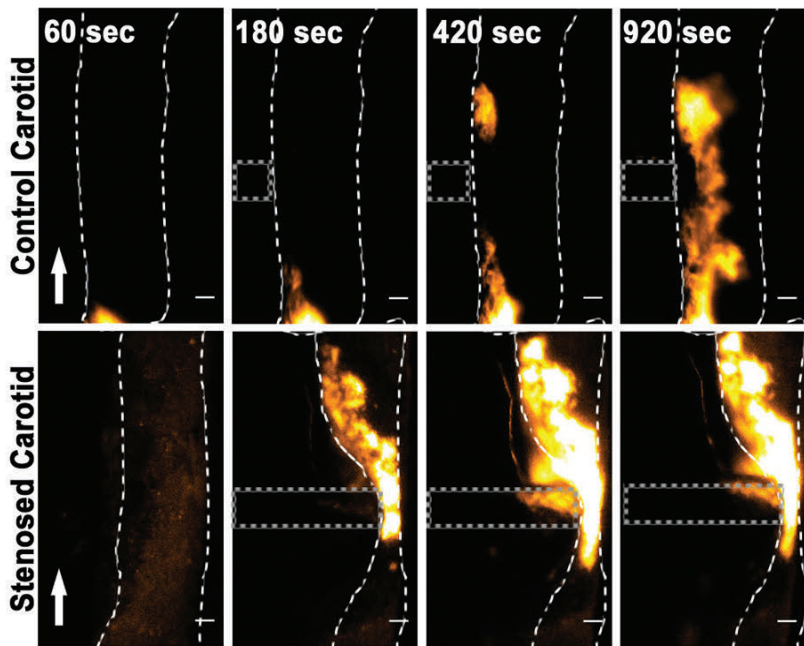

D
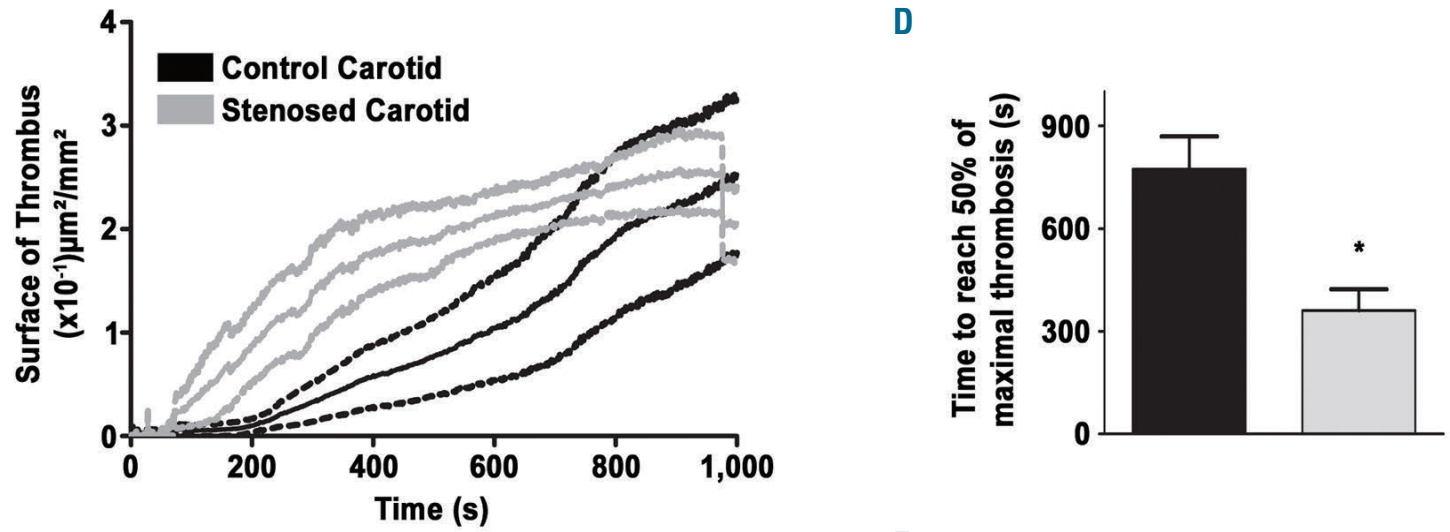

$E$

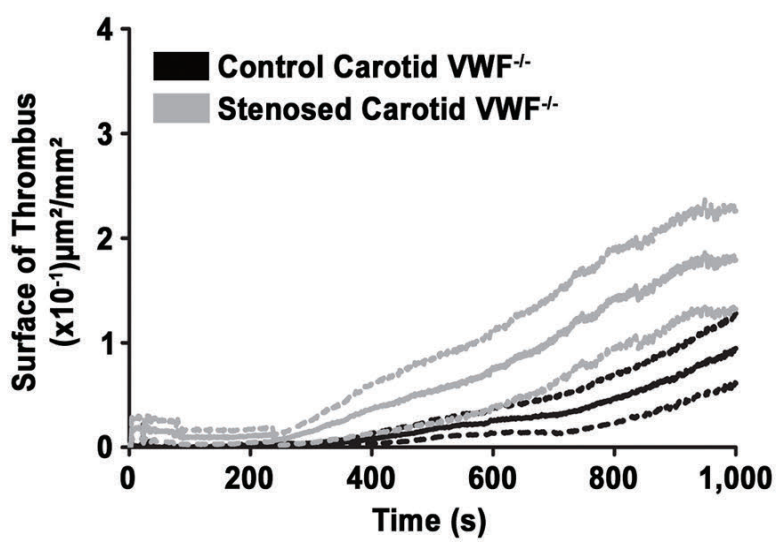

$\mathbf{F}$

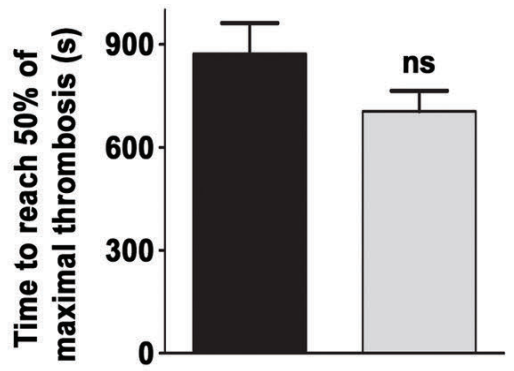

Figure 6. Thrombus growth is accelerated under shear rate gradients (SRG) in an injured mouse carotid artery. The common carotid arteries of adult mice were exposed and a filter paper saturated with $\mathrm{FeCl}_{3}$ was placed laterally on the left vessel for 2.5 minutes (min). After 4 min, the vessel was pinched with a micromanipulator to realize an $80 \%$ stenosis. (A) Computational fluid dynamic analysis presenting the wall shear rate (WSR) heat map at the middle of the stenosis carotid artery $(y=0)$ with a radius of $250 \mu \mathrm{m}$ containing a stenosis with a width of $400 \mu \mathrm{m}$ and a length of $1.35 \mu \mathrm{m}$ throughout the whole vessel. (B) Representative fluorescent images of a thrombus forming after vessel injury obtained by labeling the platelets with DiOC Scale bar: 100 um. Arrows indicate direction of blood flow. (C) Tracings representing the mean surface area ( \pm standard error of the mean [SEM], $n=9$ ) of thrombus growth after application of $\mathrm{FeCl}_{3}$ in the presence or absence of a stenosis. (D) Bar graph represents the time needed to obtain half maximal thrombus area. The shading corresponds to the SEM. Data were compared by 2 -tailed MannWhitney tests, $* P<0.05$. (E) Tracings representing the mean surface area $( \pm \mathrm{SEM}, \mathrm{n}=5)$ of thrombus growth after application of FeCl $\mathrm{F}_{3}$ and stenosis on a carotid of VWF mice. (F) Bar graph represents the time in seconds (s) needed to obtain half maximal thrombus area. The shading corresponds to the SEM. Data were compared by 2-tailed Mann-Whitney tests, $P>0.05$. 
result confirms that VWF plays a central role in the accelerated thrombosis that occurs under conditions of blood flow acceleration and SRG.

\section{Discussion}

In this study, we describe and characterize a process of bi-phasic thrombus formation on weak adhesive proteins such as fibrinogen taking place under blood flow acceleration which can be found in the vicinity of an atherosclerotic plaque. This process is original and differs from thrombus formation in perfusion assays occurring under physiological or pathological hemodynamic conditions by several features including: i) its occurrence on several weak adhesive surfaces such as fibrinogen, laminins or thrombospondin-1 which are well-known to support only single platelet attachment; ii) its bi-phasic nature with a first step of slow platelet recruitment followed by a second phase of very rapid platelet aggregation; iii) its high reactivity that results in rapid channel occlusion; iv) its crucial dependence on VWF, which is not only key in the first step of platelet recruitment to the surface, but is also required to initiate the second step of platelet aggregation through VWF fiber formation on pre-adherent platelets. The elevated reactivity of the thrombotic process occurring under SRG, was not only evidenced by the ability of platelets to adhere at high shear, but also by their increased activation, their pro-coagulant activity and by fibrin deposition resulting in occlusive thrombosis. This SRG-enhanced thrombus growth was also evidenced in vivo, after mechanically inducing a severe stenosis of a $\mathrm{FeCl}_{3}$-injured carotid artery. Our results are in line with the pioneer work of Nesbitt and co-authors who showed that shear rate gradients promote thrombus formation. ${ }^{2}$ While they proposed that this mechanism was largely independent of soluble agonists, we propose that the formation of highly reactive VWF fibers could promote enough activation to support platelet aggregation.

Our results are in line with previous reports ${ }^{7}$ showing that under normal blood flow conditions, immobilized fibrinogen recruits single platelets under low WSR with a threshold around $1,000 \mathrm{~s}^{-1}$ above which platelets fail to attach. ${ }^{7}$ The reason for the lack of attachment above 1,000 $\mathrm{s}^{-1}$ relies in the limited affinity of integrin $\alpha \mathrm{IIb} \beta 3$ for its ligand. ${ }^{7}$ We were therefore surprised by the capacity of platelets to attach to immobilized fibrinogen at a high shear $\left(4,800 \mathrm{~s}^{-1}\right)$ in the restricted area of a $90 \%$ stenosed channel. This response is not due to the local square geometry of the microfluidic device (zone 3), since it is not observed in a straight channel with similar dimensions. Instead, this effect is most likely the result of profoundly altered flow conditions resulting from the stenosis which generates SRG. Numerical studies indicated that the SRG found in the transition from zone 2 to zone 3 favor unfolding of VWF multimers, a phenomenon known to increase its adsorption to adhesive substrates. ${ }^{9}$ This likely explains the significant increase of VWF binding to fibrinogen that we observed in zone 3. Moreover, VWF unfolding dissociates the A1/A2 region and thereby unblocks the binding site of GPIb-IX complex which explains platelet recruitment to immobilized fibrinogen observed in zone 3 at $4,800 \mathrm{~s}^{-1} .^{21}$

A major observation of this work is the description of a physical and direct interaction between fibrinogen and
VWF in a purified system. We show that VWF binds to fibrinogen both under static and flow conditions, however, the binding is markedly increased in conditions of SRG, which could be explained by an increased VWF unfolding. The reason why this interaction has been overlooked so far most likely relies in the fact that in the absence of SRG, the amount of fibrinogen-bound VWF is insufficient to support platelet recruitment at high shear. We observed that under SRG the amount of VWF adsorption to fibrinogen increases (zone 3), which explains platelet recruitment at high shear. The underlying mechanism probably relies in SRG-mediated VWF unfolding which favors its adsorption. Moreover, we propose that the streamline contraction effects taking place after platelet adhesion may participate as these effects favor VWF transport to the surface. We also observed that red blood cells modestly contribute to this process, potentially by shear diffusion-mediated transport of VWF.

A striking result of this work is the very rapid platelet aggregation occurring on platelet monolayers under SRG. Real-time video-microscopy showed that this event was concomitant to the formation of VWF fibers of several hundred microns long, which could represent a trigger. This was shown in vitro, since blockade of the GPIb/VWF interaction once the monolayer had already formed, completely prevented the rapid platelet aggregation process. Similar results were obtained in vivo as the acceleration of thrombosis occurring under SRG was also markedly reduced in VWF-deficient mice. Interestingly, the fibers did not form directly on the surface of the channels, but rather at a distance on top of adherent platelets or small aggregates. The reason why VWF fibers formed in the restricted region is the result of a combination of factors. Firstly, this process occurred under SRG which efficiently promote VWF unfolding that is a prerequisite for VWF self-association and fiber formation. ${ }^{14}$ Secondly, fiber formation took place several microns above the surface where numerical studies of the flow surrounding adherent platelets predicted extremely high shear rates $\left(>10,000 \mathrm{~s}^{-1}\right)$ which also favors VWF unfolding and fiber formation. Thirdly, simulation of VWF dynamics confirmed that VWF unfolding was maximal at 10 microns above the surface. Finally, CFD indicates streamline contraction effects at such sites which facilitate recruitment of plasma VWF and therefore fiber formation.

This study identified a process of bi-phasic thrombosis on weak adhesive surfaces. Such a process was previously reported on fibrillar collagen in a stenosed channel. ${ }^{22}$ In both cases the bi-phasic process occurred when the blood flow is accelerated. Acceleration is found throughout the vasculature due to the pulsatile nature of the blood flow and the complex geometry of the vessels. However, the bi-phasic thrombosis leading to vessel occlusion described herein was achieved only for elevated values of SRG. Interestingly, despite simple hemodynamic conditions in our microfluidic system (laminar and constant blood flow), the disturbance of flow caused by the stenosis results in a complex phenomena, including accelerated thrombosis. Therefore, we propose that this process observed in the microfluidic-scale system is most likely relevant to pathological flows which can be found in the vicinity of an evolved atherosclerotic plaque presenting a severe stenosis. This has been shown by the accelerated thrombosis that occurred in an injured carotid artery when a $80 \%$ stenosis was applied with a micromanipula- 


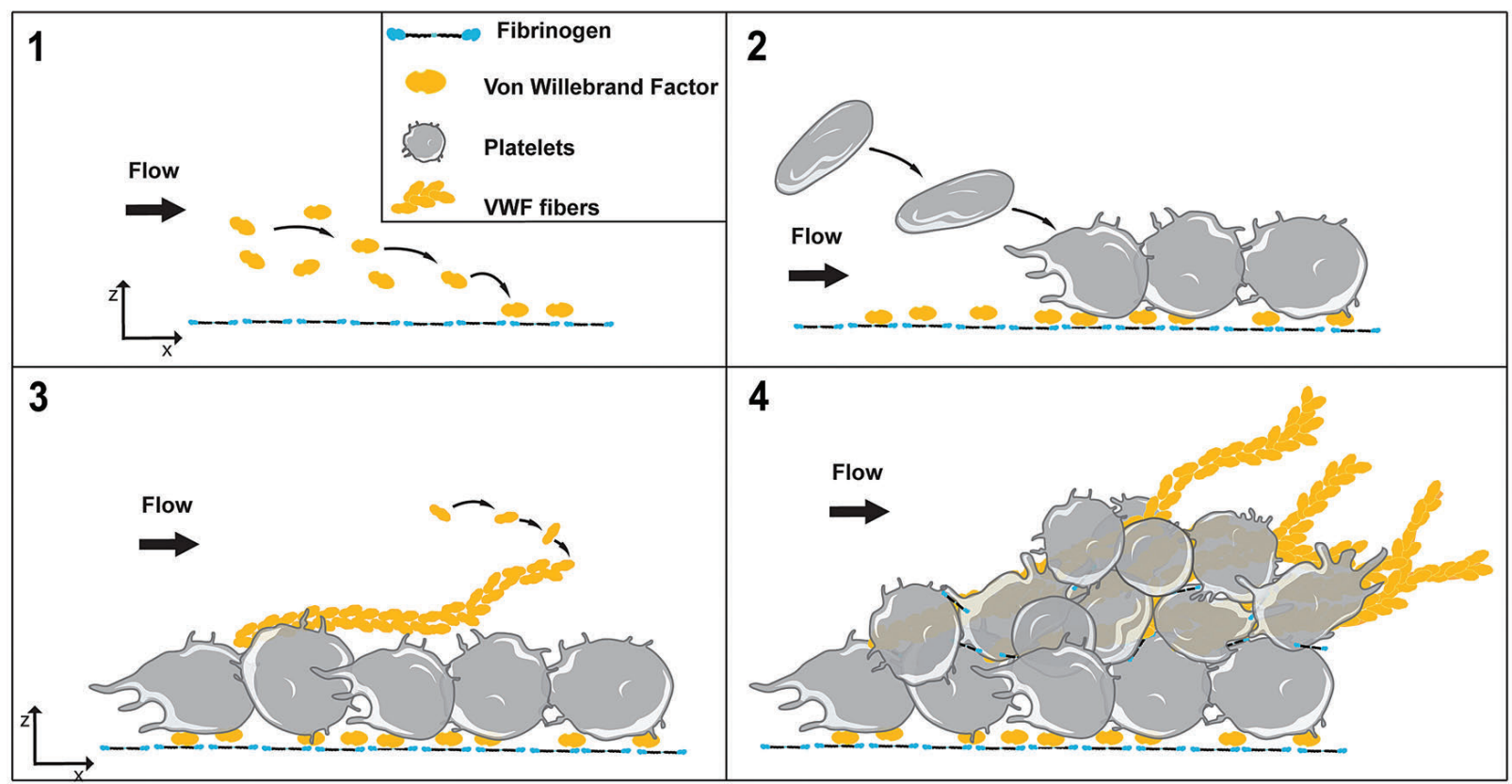

Figure 7. Model of bi-phasic thrombus formation under shear rate gradients. Shear rate gradients (SRG) found at the site of vessel stenosis favor the adsorption of plasma von Willebrand factor (VWF) on immobilized weak adhesive proteins such as fibrinogen (1). This process allows recruitment of circulating platelets at elevated wall shear rates (2). VWF fibers are then forming on a monolayer of adherent platelets at distance of the surface (3). The VWF fibers initiate rapid platelet aggregation which can lead to occlusive thrombosis (4).

tor. Whether such thrombi contain large VWF fibers and whether such fibers represent a major trigger for occlusive thrombosis still needs to be demonstrated. Based on our observations it is tempting to speculate that blockade of VWF fiber formation could efficiently reduce the accelerated thrombosis occurring when the flow accelerates. In agreement with this view, it has recently been proposed that blockade of VWF self-association could represent a potential strategy to prevent arterial thrombosis, highlighting the therapeutic potential of interfering with SRG driven thrombosis. ${ }^{23}$ Marked flow acceleration also takes place in extracorporeal circulation systems notably at the site of tubing-connector junctions where sharp $90^{\circ}$ angles are particularly prone to generate elevated SRG. ${ }^{24}$ Such devices were shown to adsorb plasma proteins including fibrinogen and to expose a surface to becoming thrombogenic under SRG, therefore explaining the occurrence of large thrombi notably in extracorporeal membrane oxygenation systems. ${ }^{24}$ Future studies should be conducted to determine the structure of these thrombi and evaluate whether they contain VWF fibers.

In summary, we describe and characterize a mechanism of bi-phasic thrombus formation that is initiated on immobilized fibrinogen, relies on VWF and occurs specifically under SRG. This process is general as it can be triggered on several weak adhesive surfaces, such as fibrinogen, laminins or thrombospondin-1, which become thrombogenic when they are subjected to blood flow acceleration generating SRG and elongational flow. Under such hemodynamic conditions, plasma VWF molecules become adsorbed to the surface allowing a modest recruitment of circulating platelets. This process is amplified on adherent platelets forming reactive VWF fibers that enhance platelet adhesion and activation, promote the procoagulant function of platelets, allow fibrin deposition and result in intense platelet aggregation and occlusive thrombosis (Figure 7).

\section{Funding}

This work was supported by ARMESA (Association de Recherche et Développement en Médecine et Santé Publique).

\section{Acknowledgments}

We would like to thank Christophe Dubois for providing the anti-fibrin antibody.

\section{References}

1. Nesbitt WS, Mangin PH, Salem HH, Jackson SP. The impact of blood rheology on the molecular and cellular events underlying arterial thrombosis. J Mol Med (Berl). 2006; 84(12):989-995.

2. Nesbitt WS, Westein E, Tovar-Lopez FJ, et al. A shear gradient-dependent platelet aggregation mechanism drives thrombus forma- tion. Nat Med. 2009;15(6):665-673.

3. Westein E, Van Der Meer AD, Kuijpers MJE Frimat JP, Van Den Berg A, Heemskerk JWM. Atherosclerotic geometries exacerbate pathological thrombus formation poststenosis in a von Willebrand factor-dependent manner. Proc Natl Acad Sci U S A. 2013;110(4):1357-1362.

4. Bini A, Fenoglio JJJ, Mesa-tejada R, Kudryk B, Kaplan KL. Identification and distribution of use of monoclonal antibodies.
Arteriosclerosis. 1989;9(1):109.

5. Ruggeri ZM. Von Willebrand factor: looking back and looking forward. Thromb Haemost. 2007;98(1):55-62.

6. Bergmeier W, Hynes RO. Extracellular matrix proteins in hemostasis and thrombosis. Cold Spring Harb Perspect Biol 2012;4(2):a005132.

7. Savage B, Saldivar E, Ruggeri ZM. Initiation of platelet adhesion by arrest onto fibrinogen or translocation on von Willebrand fac- 
tor Cell , 1996:84:289-297.

8. Siedlecki CA, Lestini BJ, Kottke-Marchant KK, Eppell SJ, Wilson DL, Marchant RE. Shear-dependent changes in the three-dimensional structure of human von Willebrand factor. Blood. 1996;88(8):2939-2950.

9. Schneider SW, Nuschele S, Wixforth A, et al. Shear-induced unfolding triggers adhesion of von Willebrand factor fibers. Proc Natl Acad Sci U S A. 2007:104(19):7899-7903.

10. Barg A, Ossig R, Goerge T, et al. Soluble plasma-derived von Willebrand factor assembles to a haemostatically active filamentous network. Thromb Haemost. 2007;97(4):514-526.

11. Colace T V., Diamond SL. Direct observation of von Willebrand factor elongation and fiber formation on collagen during acute whole blood exposure to pathological flow. Arterioscler Thromb Vasc Biol. 2013; 33(1):105-113.

12. Fu H, Jiang $\mathrm{Y}$, Yang D, Scheiflinger F, Wong WP, Springer TA. Flow-induced elongation of von Willebrand factor precedes tensiondependent activation. Nat Commun. 2017;8(1):324
13. Savage B, Sixma JJ, Ruggeri ZM. Functional self-association of von Willebrand factor during platelet adhesion under flow. Proc Natl Acad Sci USA . 2002;99:425-430.

14. Sing CE, Alexander-Katz A. Elongational flow induces the unfolding of von willebrand factor at physiological flow rates. Biophys J. 2010;98(9):L35-L37.

15. Bennett JS. Platelet-fibrinogen interactions. Ann N Y Acad Sci. 2001:936(1):340-354

16. McDonald JC, Duffy DC, Anderson JR, et al. Fabrication of microfluidic systems in poly(dimethylsiloxane). Electrophoresis. 2000;21(1):27-40

17. Eckly A, Hechler B, Freund M, et al. Mechanisms underlying $\mathrm{FeCl}$-induced arterial thrombosis. J Thromb Haemost. 2011;9(4):779-789.

18. Kurz KD, Main BW, Sandusky GE. Rat model of arterial thrombosis induced by ferric chloride. Thromb Res. 1990;60(4):269280.

19. Léon C, Eckly A, Hechler B, et al Megakaryocyte-restricted MYH9 inactivation dramatically affects hemostasis while preserving platelet aggregation and secre- tion. Blood. 2007;110(9):3183-3191.

20. Bahrami M, Yovanovich MM, Culham JR Pressure drop of fully-developed, laminar flow in microchannel of arbitrary cross-section. J Fluids Eng Trans ASME. 2006; 128(5):1036-1044.

21. Aponte-Santamaría C, Huck V, Posch S, et al. Force-sensitive autoinhibition of the von willebrand factor is mediated by interdomain interactions. Biophys J. 2015 108(9):2312-2321.

22. Bark DL, Para AN, Ku DN. Correlation of thrombosis growth rate to pathological wall shear rate during platelet accumulation Bark - 2012 - Biotechnology and Bioengineering - Wiley Online Library. Biotechnol Bioeng. 2012.

23. Chung DW, Chen J, Ling M, et al. High-density lipoprotein modulates thrombosis by preventing von Willebrand factor self-association and subsequent platelet adhesion. Blood. 2016;127(5):637-645

24. Hastings SM, Ku DN, Wagoner S, Maher KO, Deshpande S. Sources of circuit thrombosis in pediatric extracorporeal membrane oxygenation. ASAIO J. 2017;63(1):86-92. 